\title{
Influence of a periodized circuit training protocol on intermuscular adipose tissue of patients with knee osteoarthritis: protocol for a randomized controlled trial
}

Aline Castilho de Almeida', Maria Gabriela Pedroso', Jessica Bianca Aily', Glaucia Helena Gonçalves', Carlos Marcelo Pastre ${ }^{2}$ and Stela Marcia Mattiello ${ }^{1 *}$

\begin{abstract}
Background: The objective of this study is to analyze the influence of a 14-week periodized circuit training protocol on patients with knee osteoarthritis $(\mathrm{OA})$, in randomized intervention groups, on thigh intermuscular adipose tissue (interMAT), body composition, systemic inflammation, cartilage degradation, and its repercussion on pain, functional performance and quality of life.

Methods: This study presents a protocol for a randomized controlled trial. Sixty selected participants diagnosed with knee OA grades II and III, 40-65 years old and $\mathrm{BMI}<30 \mathrm{~kg} / \mathrm{m}^{2}$, will be randomly divided into three groups:periodized circuit training, strength training, and educational protocol. The circuit training and strength training protocols consist of 14-week training protocols conducted 3 times a week. The circuit training group will perform selected exercises previously stratified as light, moderate, and intense, arranged progressively in a circuit model, the strength group will perform regular strength exercises, and the educational protocol group will participate in a 14-week protocol with lectures twice a month about healthy lifestyles. Baseline and follow-up evaluations will be conducted for thigh interMAT (computed tomography), body composition (DXA), inflammation (IL-1ß, IL-6, IL-10, TNF-a, leptin, and adiponectin), and joint degradation biomarkers (UCTX-II and sCOMP), performance-based tests (30s Chair Stand Test, $40 \mathrm{~m}$ Fast-paced Walk Test and Stair Climb Test), quadriceps and hamstring maximal isometric voluntary contraction (MIVC), and questionnaires (WOMAC and pain catastrophizing scale). Repeated measures ANOVA will be used to compare differences between groups (circuit training $X$ strength training $X$ educational protocol) at the different times of assessment (baseline $x$ follow-up or baseline $x$ during protocol $x$ follow-up) for each of the dependent variables. When significant main effects were found, the pots hoc Bonferroni test will be used to identify statistical differences. A significance level of $5 \%(p<0.05)$ will be adopted.
\end{abstract}

Discussion: This will be the first randomized controlled trial to assess the effects of a circuit training protocol on patients with knee OA on thigh intermuscular adipose tissue (interMAT). Given the prevalence and impact of OA and the widespread availability of this intervention, assessing the efficacy of a low-cost, non-pharmacological, and non-invasive treatment for knee OA patients has the potential for immediate and high clinical impact.

Trial registration: ClinicalTrials.gov, NCT02761590, registered in May 4, 2016.

Keywords: Intermuscular fat, Circuit workouts, Adipokines, Quadriceps, Inflammation, CT scan, Physical therapy, Rehabilitation

\footnotetext{
* Correspondence: stela@ufscar.br

'Department of Physical Therapy, Federal University of São Carlos (UFSCar),

Km 235,Rod. Washington Luís-SP310, Postal code, São Carlos, (SP) 13565-905,

Brazil

Full list of author information is available at the end of the article
}

(c) The Author(s). 2018 Open Access This article is distributed under the terms of the Creative Commons Attribution 4.0 International License (http://creativecommons.org/licenses/by/4.0/), which permits unrestricted use, distribution, and reproduction in any medium, provided you give appropriate credit to the original author(s) and the source, provide a link to the Creative Commons license, and indicate if changes were made. The Creative Commons Public Domain Dedication waiver (http://creativecommons.org/publicdomain/zero/1.0/) applies to the data made available in this article, unless otherwise stated. 


\section{Background}

Knee osteoarthritis (OA) is a chronic inflammatory degenerative joint disease that commonly causes pain and limits activity, placing a significant burden on healthcare services [1-3]. Changes in body composition such as a decrease in muscle mass associated with an increase in adipose tissue are characteristics that can contribute to OA progression. Moreover, fat infiltration can change the orientation of muscle fibers, thereby reducing the power production capacity $[4,5]$.

Higher amounts of intermuscular adipose tissue (interMAT) in the thighs of patients with knee OA has been considered clinically significant due to the negative associations with knee extensor strength, physical function, and the systemic inflammatory process [6-8]. InterMAT can be considered a deposit of ectopic fat similar to visceral adipose tissue, which can release proinflammatory cytokines [9].

Modulation of the inflammation present in OA has been related to the occurrence of some cytokines, especially interleukin-1 $\beta$ (IL-1 $\beta$ ), interleukin-6 (IL-6), and tumor necrosis factor- $\alpha$ (TNF- $\alpha)$, which are critical mediators of metabolic disturbance and increased catabolism of joint tissues [10], and may lead to progressive loss of muscle mass [11] and interfere with cartilage degradation [12]. Adipocytes release adipokines such as leptin and adiponectin, which can cause and exacerbate chronic low-level systemic inflammation. Studies suggest that leptin may act to regulate chondrocyte metabolism and has been related to the metabolic (non-mechanical load) effect of obesity on joint disease and could explain the association between joint disease and metabolic syndrome disorders $[10,13,14]$. Adiponectin has also been proposed as a systemic biomarker of OA. Plasma adiponectin was significantly higher in a population of $\mathrm{OA}$ patients [11]. In muscle, adiponectin has been shown to increase fatty acid oxidation and glucose uptake, and to attenuate local inflammation [15].

The catabolic processes of articular cartilage evaluation present in OA are very important for better understanding of its pathogenesis, as well as being a method to evaluate its development in the short term. Biomarkers, such as uCTX-II and COMP, have the capacity to detect early joint degradation in degenerative diseases such as OA [16], being, therefore, significantly associated with the incidence and progression of OA [17].

Although a number of biochemical markers of joint tissue turnover have been developed and tested, the majority of studies have been limited to cross-sectional studies of people with and without OA or observational studies of OA progression [18]. It has been described in the literature that physical exercise modifies the inflammatory condition of the body, especially by modifying the circulation of IL-6 [19] and increasing anti-inflammatory cytokines such as IL-10 [20]. Thus, these cytokines are related to basic morbidities under the influence of different types of exercises.

Considering the reduction in physical activity and quality of life found in individuals with knee OA (due to the influence of inflammation and changes in body composition) [21], non-pharmacological, conservative clinical guidelines are encouraged, and physical exercises are recommended due to their beneficial effects, low potential for adverse effects, and low cost [22-24].

Studies assessing the effects of exercise on knee OA are widespread and are intended to reduce pain, and improve muscle strength, stability of joints, and aerobic fitness, leading to improvement in functionality $[24,25]$. Although several studies have demonstrated its effectiveness in increasing strength, reducing pain, and improving functionality [26], there is no evidence of the effect of exercise on thigh composition (adipose tissue and muscle mass) and the related impairment in individuals with knee OA.

It is known that physical exercise can increase muscle mass and muscle strength [27] and has shown promise in protecting the knee joint and reducing inflammatory cytokines [28, 29]. In a systematic review, Juhl et al. (2014) investigated training protocols used to treat patients with knee OA and reported that optimal exercise programs for this population should focus on improving aerobic capacity, quadriceps strength, and lower limb performance [25]. However, Bennell and Hinman (2011) reported that a combination of both resistive and aerobic training is better suited to deal with the various complications associated with OA [23]. Similarly, Nguyen et al. (2016), in a review study related to the efficacy and safety of exercise therapy and strength training, reported that although rehabilitation is a key treatment in $\mathrm{OA}$ and widely recommended, the ideal method for therapy programs remains inconsistent [30]. In this way, enriching the reasons for exercise prescription seems to be a gap that needs filling.

One way to combine the benefits of these modalities is circuit training, used to stimulate both systems, which promotes cardiovascular and muscle strengthening [31, 32]. The effects of circuit training on several chronic disorders are known and positive effects have been shown on body composition by reducing body fat, which can be achieved by a repeated series of exercises, with little or no rest interval between them [33]. This type of exercise can more effectively activate lipolysis of adipose tissue than conventional aerobic training $[31,34]$. This allows many people to participate in the same training session due to low total duration of the exercises [31, $33,35,36]$, which promotes high retention and adherence of participants [37]. 
Despite having recently attracted the interest of researchers due to the potential benefits that this technique provides, the effects of a periodized circuit training protocol on interMAT remain unclear. Only three studies in the consulted literature have evaluated the effects of circuit training on knee OA patients. These studies showed a reduction in body mass and adipose tissue (assessed by dual X-Ray absorptiometer (DXA)), increase in muscle strength, improved knee function, and a reduction in pain compared with a control condition [38-40]. However, the training protocol did not follow a periodized model.

Adipose tissue concentration and muscle quality are directly linked with physical activity level and exercise intensity. Few studies have explored the physical activity level of their sample [41-43] and used it to normalize or discuss data. In older adults, a greater physical activity level is associated with less visceral adipose tissue, subcutaneous fat, and interMAT [44].

To date, no studies have assessed the changes in muscle fatty infiltration in the thigh after exercise and its implications, such as inflammation levels in the knee OA population. There is evidence that exercise in combination with diet is effective for decreasing total body fat mass, inflammation levels, and pain, in addition to improving physical function in overweight and obese adults with knee OA [45]. In overweight and obese adults, resistance training improved body composition, including reduction in thigh interMAT [46]. Meanwhile, in older fallers, there were no significant changes in thigh interMAT content after 3 months of resistance training [47]. It seems that exercise can decrease fat infiltration in thigh muscles of overweight and obese. However, in older fallers this was not seen.

Considering the possible alterations in muscle function and morphology caused by the presence of fat tissue in thigh muscles, it is essential that new study proposals involving a periodized circuit training protocols identify not only body fat reduction, but also the local effects of the amount of interMAT.

It is understood that a randomized controlled trial, the gold standard in intervention studies [48], is the best way to evaluate new treatments for a given variable, as suggested by Messier et al. (2015) in the study recommendations, design, and conduct of clinical trials in patients with OA [49]. The present study protocol has an investigative character and we emphasize its originality, mainly because the influence of periodized circuit training on the main study variable (interMAT) is still unknown.

The results of this research may contribute to elaboration of future treatment strategies and early intervention in the causative and potentiating factors of the disease, verifying the effects of a low-cost, non-pharmacological, and non-invasive treatment to provide subsidies for better rehabilitation planning for patients with knee OA.

\section{Methods/Design \\ Aim}

The main objective of the present study will be to compare the influence of 14-weeks of periodized circuit training with strength training and an educational protocol on thigh interMAT and body composition. Secondarily, the present study will investigate these effects on indicators of cartilage degradation and systemic inflammation, and its repercussions on physical function, pain, and quality of life in patients with knee OA.

Our general hypothesis is that both circuit training and strength training protocols will promote decreases in thigh interMAT, associated with improvement in body composition and muscle strength, and a reduction in inflammatory cytokine concentration (IL1 $\beta$, IL6, TNF- $\alpha$ ), pain, and stiffness in patients with knee OA, leading to consequent improvement in quality of life, compared to the educational protocol. However, it is hypothesized that these benefits, mainly related to the improvement in the body composition and reduction in interMAT will be more evident after the circuit training, compared to the strength training. Additionally, it is also hypothesized that biomarkers of joint degradation may signal exercise efficiency for both groups over the application of both training protocols.

The following methodology is in full agreement with the SPIRIT (Standard Protocol Items for Randomized Trials) recommendations [50-52] [50-52] and the Osteoarthritis Research Society International (OARSI) recommendations for RCTs [53] to ensure methodological rigor. Consolidated Standards of Reporting Trials (CONSORT) [54] guidelines will be followed for reporting the results in a subsequent article.

\section{Study design}

The present proposal is characterized as a single-blinded randomized controlled trial design. This is the first version of the study protocol, submitted to the registry of clinical trials Clinical Trials (clinicaltrials.gov) and registered in May 4, 2016, under identification code NCT02761590.

\section{Ethical aspects}

This study was approved by the Research in Human Ethics Committee (CEP) of the Federal University of São Carlos-SP. Participants will be informed about the procedures that will be performed throughout the research, and after agreeing to participate, will sign an informed consent form (ICF). This study will be conducted according to Resolution 466/12 norms of the National Health Council on research involving humans. 


\section{Blinding}

The outcome assessor will be blind to group allocation and will not be involved in the interventions or attend any of the testing. The physical therapists supervising the exercise intervention sessions will not be blinded. The statistician will be blinded to group allocation prior to completion of the statistical analysis. With training and standard operating procedures, it is anticipated that any performance bias due to lack of blinding will be minimized.

\section{Recruitment}

Participants from the community of São Carlos, Brazil, will be invited to participate in this study. Three main methods will be used to recruit potential participants: advertisements will be placed in local newspapers, magazines, and social media. Potential participants will be invited to complete a phone interview to pre-screen their eligibility to ensure that they meet the study selection criteria prior to randomization. If deemed eligible, an appointment will be scheduled to confirm eligibility, sign the ICF, and complete baseline evaluations.

\section{Sample}

To determine the sample size, the data of thigh interMAT from the study of Griessmann et al. (2014) were considered [55]. 16 participants per group was stipulated through a two-tailed hypothesis test, with a $5 \%$ level of significance and $80 \%$ test power.

Considering the possible sample loss in the course of the study, 20 subjects each, totaling 60 participants of either sex will be included in this study.

To be eligible, participants will be required to fulfill the following criteria:

i. Aged between 40 and 65 years;

ii. KOA clinically diagnosed fulfilling the American College of Rheumatology classification criteria of knee pain on most days of the past month and radiographically, classified as grades 2 or 3 according to Kellgren and Lawrence criteria [56].

iii. Knee pain for $\geq 3$ months;

iv. Overall average knee pain in the last week $\geq 4$ on a 0 to 10 Visual Analogue Scale (VAS).

The exclusion criteria are:

i. Knee surgery;

ii. Body mass index (BMI) $\geq 30 \mathrm{~kg} / \mathrm{m}^{2}$;

iii. Previous history of lower limb trauma;

iv. Physical therapy, chiropractic, or acupuncture treatment or exercises specifically for the knee within the previous 6 months; v. Walking more than 30 min continuously daily or participating in a regular (more than twice a week) exercise program;

vi. Current or past (within 3 months) oral or intraarticular corticosteroid use;

vii. Systemic arthritic conditions such as rheumatoid arthritis;

viii.Inability to walk unaided as this is necessary for some of the physical testing;

ix. Medical condition precluding safe exercise such as uncontrolled hypertension, heart condition, cardiac pacemaker use, chronic diseases, impaired renal function, or any other medical condition that precludes participation in the study $[57,58]$;

x. History of severe muscular lesions (above grade I);

xi. Motor impairment due to neuromuscular diseases;

xii. Cognitive deficits that compromise understanding of the tests;

xiii.Starting any other exercise protocol or physical therapy during the study.

xiv. Unable to comply with the protocol such as inability to attend therapy session or attend assessment appointment at the University.

xv. Kellgren and Lawrence grades 0, 1, and 4 .

\section{Procedures}

All experimental procedures will be performed following the timeline shown in Fig. 1. The evaluations will be conducted at the Articular Function Analysis Laboratory (LAFAr) and the training protocols will be conducted at the Physical Therapy Department of the Federal University of São Carlos (UFSCar), in a room with a controlled temperature (between $21{ }^{\circ} \mathrm{C}$ and $23^{\circ} \mathrm{C}$ ).

On day 1, participants will be guided and familiarized with the experimental procedures to be performed. An evaluation form (personal and anthropometric data, blood pressure, knee pain, and previous medical history) and the informed consent form will be completed. During the same visit, the body composition test (Dual Energy X-ray Absorptiometry - DXA) will be performed and the participant will receive a referral for the knee radiographic exams.

Day 2 will be scheduled after the OA radiographic diagnosis and classification. During this visit, the WOMAC (Western Ontario and McMaster Universities) and Pain Catastrophizing Scale (PCS) questionnaires will be completed. Subsequently, the participants will perform three functional performance-based tests $(40 \mathrm{~m}$ fast-paced walk test, $30 \mathrm{~s}$ chair stand test, and stair climb test), and the strength test. The functional performance tests and the strength test will be performed in random order. Participants will then receive the referral and guidelines for the computed tomography exam for thigh composition (adipose tissue and muscle mass) analysis 


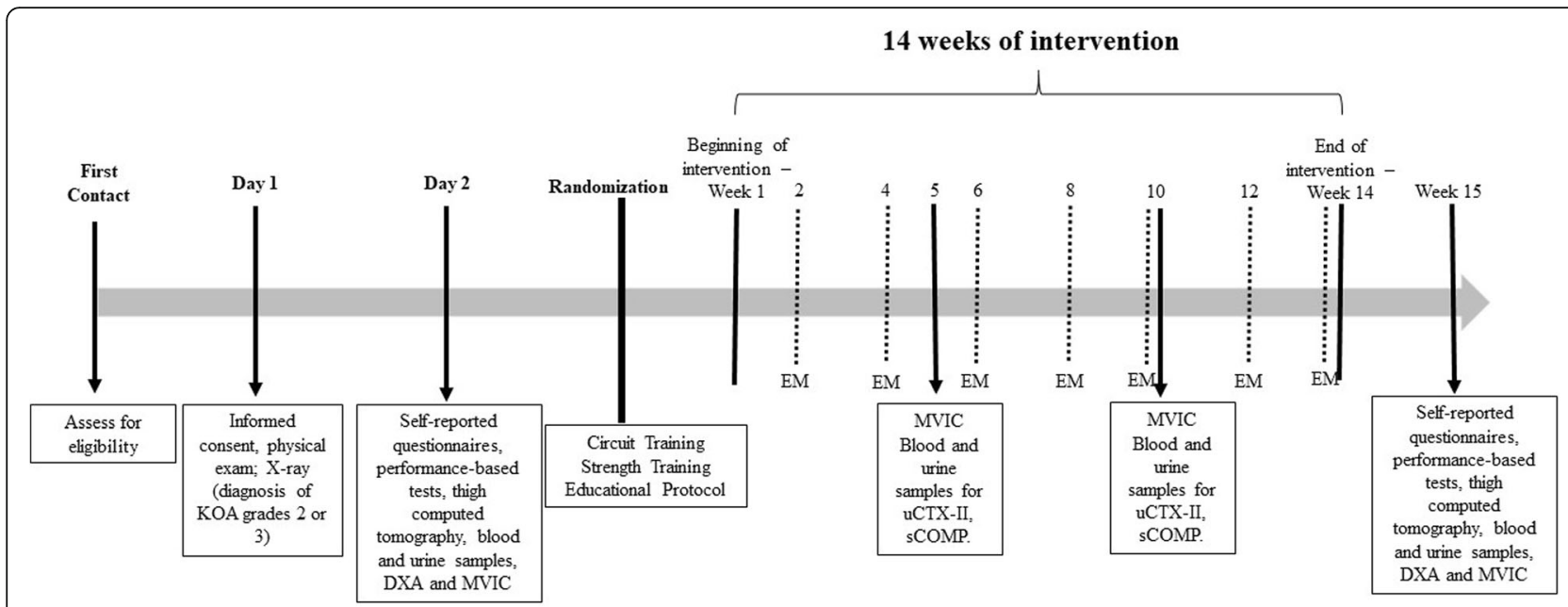

Baseline

Follow-up

Fig. 1 Timeline of study procedure; EM: Educational meeting sessions; DXA: dual X-Ray absorptiometry; MVIC: maximal voluntary isometric contraction

and blood and urine samples will be collected for inflammatory and collagen activity marker analysis, identifying the systemic responses to exercise. After the baseline evaluations (Days 1 and 2), participants will be randomized through a randomization website (https:// www.random.org/) into three groups: periodized circuit training, strength training, and educational protocol. The group distribution will be balanced for: number of males and females, age, body mass index (BMI), and radiographic severity. A researcher not involved with the evaluations and intervention sessions will be in charge of conducting the participant randomization and balance distribution. During and at the end of the 14 weeks intervention protocols, follow-up evaluations will be scheduled (Fig. 1).

Data collections will be performed over the 14 weeks of the study. At baseline and post 14 weeks intervention (follow up) the following will be performed: computed tomography of thighs, maximal voluntary isometric contraction (MVIC), performance-based tests, blood and urine samples, questionnaires, and DXA. In the 5th, 10th, and 14th weeks, blood and urine samples will be collected for sCOMP and uCTX-II analysis and the MVIC test will be conducted.

All analyzes will be performed for all groups blindly, i.e., the evaluator of the collected data will be blinded to the group allocation.

\section{X-ray}

All participants will undergo radiographic exams of the knees for the diagnosis of unilateral or bilateral osteoarthritis. Bilateral posterior-anterior weight-bearing semi flexed knee $\mathrm{x}$-rays will be used to identify tibiofemoral $\mathrm{OA}$ and skyline views to identify patellofemoral OA
[59, 60]. The Kellgren and Lawrence (1956) [56] and American College of Rheumatology criteria will be considered to diagnose and classify KOA, considered as: Grade 0, without changes; Grade I, minimal osteophytes with dubious presence; Grade II/o, defined osteophytes and absence of joint space decrease; Grade II, defined osteophytes and minimal reduction in joint space, grade I in the classification of joint space reduction of Altman and Gold (2007); Grade III, presence of osteophytes and moderate reduction in joint space; Grade IV, significant reduction in joint space and subchondral bone sclerosis $[61,62]$.

\section{Thigh computed tomography}

Intermuscular adipose tissue of both thighs will be the primary outcome for this study. For this, computed tomography scans will be conducted using a Multislice Tomograph (Brilliance CT 16-slice, Phillips), located at the University Hospital of the Federal University of São Carlos. The exam will be performed by a specialized radiologist.

Image acquisition will be performed as described by Messier et al. (2013) [2]. Participants will be placed supine with their legs held in a neutral position. A 2-dimensional topogram will be obtained from the pelvis to the knees. In order to obtain the scans, the following parameters will be established: helical mode, $120 \mathrm{KV}$, $150 \mathrm{mAs}$, with reconstruction of both legs at $5 \mathrm{~mm}$ slice thickness and $50 \mathrm{~cm}$ display field of view (DFOV).

To establish the site of interest for analysis of thigh composition, the total femur length (from the greater femoral tuberosity to the lower border of the medial femoral condyle) will be transected into three parts. The junction between the proximal and mid-third will be 
marked for measurement. The area analyzes of thigh composition (interMAT, subcutaneous adipose tissue, and muscle mass) will be manually performed using ITK-SNAP (version 3.6) software, and the area of interest will be selected by a scan according to tissue density attenuation rates for quantification of adipose tissue presented in $\mathrm{cm}^{2}$. Additionally, a manual line will be made separating the bony part of the soft tissues, and then the adipose tissue present within the bone area will be subtracted from the area obtained by measuring the interMAT and subcutaneous adipose tissue. Skeletal muscle and adipose tissue areas will be calculated by the range of attenuation values for skeletal muscle (0 to $100 \mathrm{HU}$ ) and adipose $(-190$ to $-30 \mathrm{HU})$ tissue $[63,64]$. Test-retest reliability on thigh scans re-analyzed 1 week apart was ICC $=0.973$ and between evaluators was ICC $=0.986(n=20)$.

\section{Body composition}

Dual Energy X-Ray Absorptiometry (DXA, Hologic Discovery A, Bendford, MA) will be used for measurements including lean body mass, body fat mass, and bone mineral density, allowing, therefore, estimation of the total body composition and per body segment.

The scanning will be performed according to the manufacturer's recommendations. According to the instruction manual, the operator should check that no metal or plastic objects remain in the scanning area, since it may alter the attenuation ratios of the DXA energy [65]. This includes hair clips and pins, snaps, zippers and buttons, jewelry, earrings, bracelets, watches, or rings. The participants will be asked to arrive in a fasted state (at least $4 \mathrm{~h}$ ) and not to perform any physical activity for $24 \mathrm{~h}$ before the exam in order to reduce the biological variability [66].

The participant will be placed in the supine position, in the center of the scanning table, with the head just below the head line marked on the table and remain unmoving during the examination. DXA software will automatically define areas of regional body estimates (left and right arms, legs and trunk) [65]. For this study, it was defined that segment and total body composition areas will be considered to analysis.

\section{Inflammatory and degradation of articular cartilage biomarkers}

Blood samples will be collected in the early morning after a 12-h fast. In total, $4 \mathrm{ml}$ of blood will be collected from the antecubital vein of each participant using a standard procedure heparin-coated vacutainer tube by a specialized laboratory. Participants will be asked to discontinue the use of anti-inflammatories for $72 \mathrm{~h}$ prior to collection.
After collection, the blood will remain for $4 \mathrm{~h}$ at rest in the refrigerator. Subsequently it will be centrifuged at $1500 \mathrm{rpm}$ for $10 \mathrm{~min}$ and the supernatant will be stored as aliquots and frozen at $-80{ }^{\circ} \mathrm{C}$. Serum concentrations of IL-1- $\beta$, IL-6, TNF- $\alpha$, IL-10, leptin, adiponectin, and sCOMP will be measured by the ELISA method (Enzyme-Linked Immunosorbent Assay), according to the manufacturer's recommendations (Quantikine, R\&D Systems, Minneapolis, MN). Patients will be instructed to perform the blood collections in the morning and remain at rest for $30 \mathrm{~min}$ [67].

To assess UCTX-II concentration, participants will be instructed not to perform physical activities for $24 \mathrm{~h}$ prior to collection, as well as which, urine will be collected in two specific containers (each with a capacity of $80 \mathrm{ml}$ ) of the first urine of the day. Participants will be instructed to fill most of the containers provided and keep the containers in the refrigerator $\left(2\right.$ to $\left.8{ }^{\circ} \mathrm{C}\right)$ until delivery to the researcher, which should be no more than $2 \mathrm{~h}$ after collection. One of the samples will be sent to the laboratory responsible for determining the creatinine concentration, while the other sample will be centrifuged at $3000 \mathrm{rpm}$ for $10 \mathrm{~min}$ for homogenization and stored in cryovials for freezing at $-80{ }^{\circ} \mathrm{C}$ in a freezer. The procedures will be performed according to the manufacturer's instructions (Elabscience Biotechnology, Texas, USA) to detect the uCTX-II concentration by the ELISA method.

Samples will be read in a spectrophotometer specific for microplate reading, using a $490 \mathrm{~nm}$ filter. The uCTX-II concentration values (ng/L) will be normalized by the total urine creatinine $(\mathrm{mmol} / \mathrm{L})$ as well as the correction of uCTX-II concentration unit in $\mathrm{ng} / \mathrm{mmol}[46,68]$.

\section{Questionnaires \\ WOMAC}

The WOMAC (Western Ontario and McMaster Universities) questionnaire will be used to assess pain, stiffness, and physical function. This is a self-administered instrument that addresses the impact and restrictions specifically in the quality of life of patients with lower limb OA, translated and validated to Portuguese [69]. The WOMAC contains 24 self-reported issues, based on information within $72 \mathrm{~h}$ prior to its application, divided into three domains: pain, stiffness, and physical function. Scoring is performed using a Likert scale, wherein each issue scores between 0 and 100, distributed as follows: $0=$ none; $25=$ mild; $50=$ moderate; $75=$ intense; and $100=$ very intense [69]. The final score will be determined by the maximum score for each domain; the higher the score, the worse the pain, stiffness, and physical function.

\section{Pain catastrophizing scale (PCS)}

The Pain Catastrophizing Scale is a self-administered scale, easy and fast to apply. This scale will be used to 
identify individuals with psychological characters for catastrophizing. This instrument consists of 13 items, in which the patient should report the degree of thought or feeling described in relation to pain, always respecting a graduation of 5 points. The total score is given by the sum of all items, ranging from 0 to 52 points. The patients will be asked to answer the questions according to the thoughts and feelings that develop when they are affected by pain, regardless of whether at the moment of the interview the patient is in pain [70].

\section{Performance-based tests}

The performance-based tests selected for this study follow the OARSI recommendations for specific purposes of functional capacity evaluation of patients with OA of the knee or hip [71].

$40 \mathrm{~m}$ fast-paced walk test (40 m FPWT)

This test will be conducted to evaluate a short distance walking activity and changing direction during walking. A $10 \mathrm{~m}$ walkway will be marked out with bright colored tape at each end. A cone will be placed approximately $2 \mathrm{~m}$ before the start mark and $2 \mathrm{~m}$ beyond the finish mark of the $10 \mathrm{~m}$ walkway for turning. Participants will be instructed to wear comfortable walking footwear (e.g. tennis shoes).

Participants will be asked to walk as quickly but as safely as possible, without running, along a $10 \mathrm{~m}$ walkway and then turn around a cone, return, and then repeat the walk again for a total distance of $40 \mathrm{~m} \mathrm{(3}$ turns). Regular walking aids will be allowed and recorded. Timing will start on the signal to start at the start line and terminate once the participant crosses back over the start line after completing the $40 \mathrm{~m} \mathrm{(4 \times}$ $10 \mathrm{~m})$. When the participant crosses the $10 \mathrm{~m}$ mark, timing will be paused whilst the participant turns around the cone and then resumed once they cross the $10 \mathrm{~m}$ mark again. The same will be repeated for the following turns and the timer stopped once the participant crosses the start line for the final time. The time of one trial will be recorded to the nearest 100th of a second and expressed as speed $\mathrm{m} / \mathrm{s}$ by dividing distance $(40 \mathrm{~m})$ by time (s) [71].

\section{$30 \mathrm{~s}$ chair stand test (30s-CST)}

This test will be conducted to evaluate the sit-to-stand activity, lower body strength, and dynamic balance. This test will evaluate the maximum number of chair stand repetitions possible in a $30 \mathrm{~s}$ period. The back of the chair will be placed against a wall, so the chair does not slide backwards. Participants will be instructed to wear comfortable walking footwear (e.g. tennis shoes). The same chair will be used for all participants, at baseline and follow up assessments. A straight back chair will be used with a $44 \mathrm{~cm}$ seat height, without arms.

The participants will sit on the chair in a position that allows them to place their feet flat on the floor, shoulder width apart, with knees flexed slightly more than 90 degrees so that their heels are somewhat closer to the chair than the back of their knees. Participants will be instructed to cross their arms at the wrists and hold them close to the chest (across chest). The tester will stand close to the side of the chair for safety and so they can observe the technique, ensuring that the participant reaches a full stand and full sit position during the test. A practice trial on one slow paced repetition will be performed before testing to check technique and understanding. From the sitting position, the participant will stand up completely with hips and knees fully extended, then sit completely back down, so that their bottom is in full contact with the seat. This will be repeated for $30 \mathrm{~s}$. If the person is not able to stand even once then they will be allowed to place their hands on their legs or use their regular mobility aid. This will be then scored as an adapted test score.

On the signal to begin, the stop watch will be started. The total number of chair stands (up and down equaling one stand) completed in $30 \mathrm{~s}$ will be counted. If a full stand has been completed at the end of the $30 \mathrm{~s}$ (i.e. standing fully erect or on the way down to the sitting position), then this final stand will be counted in the total. The participant will be allowed to stop and rest if they become tired, but the timer will continue. If the person is not able to stand even once then the score for the test will be zero. If the person is able to stand with adaptions, such as hands placed on their legs, then the number of stands will be recorded as an adapted test score and the adaptations made to the test indicated $[71,72]$.

\section{Stair climb test (SCT)}

This test will be conducted to evaluate ascending and descending stair activity, lower body strength, and balance, using a 12-steps flight of stairs with handrails. Each step will measure $16 \mathrm{~cm}$ in height and $30 \mathrm{~cm}$ in depth, and adequate lighting, free from traffic and external distractions will be ensured. Participants will be instructed to wear comfortable walking footwear (e.g. tennis shoes), and if safety is of concern, the tester will stand behind/below the participant, going up the stairs and ahead/to the side coming down the stairs. If there is no concern for safety, the tester will remain at the start/finish position on the ground landing. A practice trial with the tester standing in the above positions will be conducted to assess safety. 
The participant will be instructed to ascend and descend the flight of stairs as quickly as possible but in a safe manner, using the handrail only if needed (if used, this will be recorded). Timing (stop watch) will begin on the signal to start and terminate when the participant returns with both feet to the ground level. The participant can stop and rest if needed but the timer will continue. The total test duration will be timed (in seconds), with longer times indicating more compromised physical function [72].

\section{Muscle strength test - maximal voluntary isometric contraction (MVIC)}

Knee maximal voluntary isometric contraction (MVIC) extension and flexion will be measured using a handheld dynamometer (Lafayette Instruments, Lafayette, IN, USA). Prior to the tests to measure the MVIC, participants will be positioned and asked to perform sub-maximal contractions for warm-up and familiarization with the test. Quadriceps MVIC will be performed in the seated position with a hip angle of $90^{\circ}$ flexion and knee angle of $0^{\circ}$ (full extension). A Velcro strap (attached to the floor) will be wrapped around the ankle, and another strap will be used to maintain the pelvis against the table. Evaluation of the knee flexor MVIC will be performed at $15^{\circ}$ knee flexion in the supine position. A Velcro strap will be wrapped around the ankle and another to secure the pelvis against the table.

The tester will place the dynamometer at the front of the ankle under the Velcro strap to measure knee extension force, and at the back of the ankle under the Velcro strap to measure knee flexion force. Before each test, the tester will demonstrate the direction of the force required. The participant will be instructed to contract "as forcefully as possible", with a gradual increase in force and strong verbal encouragement will be provided during the contractions. The tester will maintain the dynamometer stable, to prevent it from being displaced. The participants will perform 3 MVIC separated by a 60-s pause (Selistre et al. 2017), and the highest value will be used for analysis. The MVIC will be expressed in kgf [73]. The reliability of the isometric muscle tests with a handheld dynamometer has been reported previously. Studies demonstrated excellent concurrent validity compared with an isokinetic dynamometer and also excellent inter-tester and intra-tester reliabilities for measuring maximal isometric strength in the main movements of lower limbs [74-77]. Additionally, in a pilot study with 10 participants with the same sample characteristics, a two-way mixed model was used to calculate the intraclass correlation coefficient (ICC) within trials. The average measure with 95\% confidence interval (CI) and coefficient of variation $(\mathrm{CV})$ were calculated in order to demonstrate the reliability of the mean of recordings.
The ICC values of MVIC were higher than 0.80 , ranging from 0.88 to 0.98 . The $\mathrm{CV}$ values ranged from 11 to $32 \%$.

\section{Muscle quality}

Muscle quality is the intrinsic ability of muscle tissue to produce force, which will be estimated through the ratio between the muscle strength, obtained by MVIC, and lean mass of the thigh, obtained by computed tomography $\left(\mathrm{kgf} / \mathrm{cm}^{2}\right)$ [78].

\section{Periodized circuit training protocol (CT) Selected exercises}

For elaboration of the periodized circuit training protocol, specific exercises for the knee OA population were selected from previously published studies [79-81].

These exercises were previously arranged in a circuit model: upper body (UB), lower body (LB), and trunk and global exercises (which involve the whole body). A total of 47 exercises were selected (Additional file 1) and then stratified in a pilot study according to the stress intensity levels; light, moderate, and intense. For the stratification, all exercises were performed by 10 subjects with similar characteristics to this study sample (age, demographics, and clinical condition). All participants in the pilot study used a heart rate monitor (Polar V800, Polar Electro Oy, Finland) to obtain HR and the Rating of Perceived Exertion (RPE) was evaluated through the Borg scale with 6-20 points, before and immediately after each exercise. Subsequently, the percentage of the maximum heart rate (\%HRmax) was calculated, by subtracting 220 from the participant's age [82]. For stratification, the Muyor (2013) study was considered, with Rating of Perceived Exertion (RPE) values 6-10: mild; 11-14 moderate; 15-20 intense; and \%HRmax values: < 54\% light; 55-69\% moderate; $>70 \%$ intense [82].

The 47 exercises selected were divided into three days (20 per day), with a 48-h interval between them. The participants were instructed to perform each exercise for $30 \mathrm{~s}$, as fast as possible, and a rest interval was given between exercises, considered sufficient for HR to return to pre-exercise values. The interval time ranged from 1 to $3 \mathrm{~min}$. Therefore, with the results of the pilot study, it was possible to organize these exercises through the 14-week training protocol, respecting the intensity progression (light, moderate, and intense).

During the training protocol, it was determined that the exercises stratified as mild will last $20 \mathrm{~s}$, moderate exercises will last $30 \mathrm{~s}$, and the intense exercises for $40 \mathrm{~s}$. Between each station, as described above, there will be a maximum of $30 \mathrm{~s}$ rest. This strategy will be used to require the contraction of several muscle groups, with a short rest between stations, causing cardiovascular and metabolic benefits and greater reduction in adipose 
tissue [83]. It should also be noted that this technique, in addition to resulting in a lower total exercise time, maximizes the impact of an exercise protocol in a short time.

\section{Application of the periodized circuit training protocol}

The circuit training protocol will be conducted in three sessions per week for 14 weeks, totaling 42 exercise sessions. The minimum percentage of sessions that the participants is required to attend is $75 \%$ of the total sessions. Each session will consist of: warm-up exercises for $5 \mathrm{~min}, \mathrm{CT}$, and cool-down for $5 \mathrm{~min}$ with global stretching exercises. Initially, participants will be asked for their perception of pain or discomfort through a visual analogue scale (VAS) and will be instructed to inform the location of the pain through the Nordic questionnaire musculoskeletal symptoms; baseline blood pressure (BPi) and initial heart rate ( $\mathrm{HRi}$ ) will also be measured. Subsequently, the participants will perform a warm-up exercise for $5 \mathrm{~min}$ on a stationary bicycle with minimum resistance.

Immediately after the warm-up exercise, each participant will perform the circuit training protocol. The participants will be instructed to perform each exercise as fast as possible, with the maximum number of repetitions during the established time in each training phase (light, moderate, and intense). The first station of exercises to be performed will be defined in a random order at each session. After the end of the first exercise station, the participant will move to the next exercise station, following a specific order to allow different muscle groups to alternate between rest and work, favoring recovery and minimizing the risk of muscle fatigue [34] as shown in Fig. 2.

Periodized dynamic intensity is a strategy used to define the dynamic intensity according to weeks of training (Fig. 3). The volume of work is defined by the training session duration and through the previously stratified intensity of effort and heart rate response to exercise, as described above.

The construction of the periodization model to be used, regarding the biological principle of volume vs. intensity interdependence, and a duration of 14 weeks, proposes a week of recuperative exercises after two weeks of stress, gradually increasing the intensity with respective volume settings. This model is based on the concepts described by Turner (2011) which concludes in favor of the organization of training adapted to the reality of the public to be trained.

The representation in Fig. 3 can be detailed as follows: the yellow column represents the adaptation and familiarization week (1st week) in which participants will be familiarized with the materials and exercises performed during the training. The exercises previously stratified as light will be performed in the second, third, and fifth weeks of training (green columns), moderate exercises will be performed in the sixth, eighth, and ninth weeks (purple columns), and intense exercises will be performed in the eleventh, twelfth, and fourteenth weeks (red columns).

Exercise duration

( 20,30 or 40 seconds)

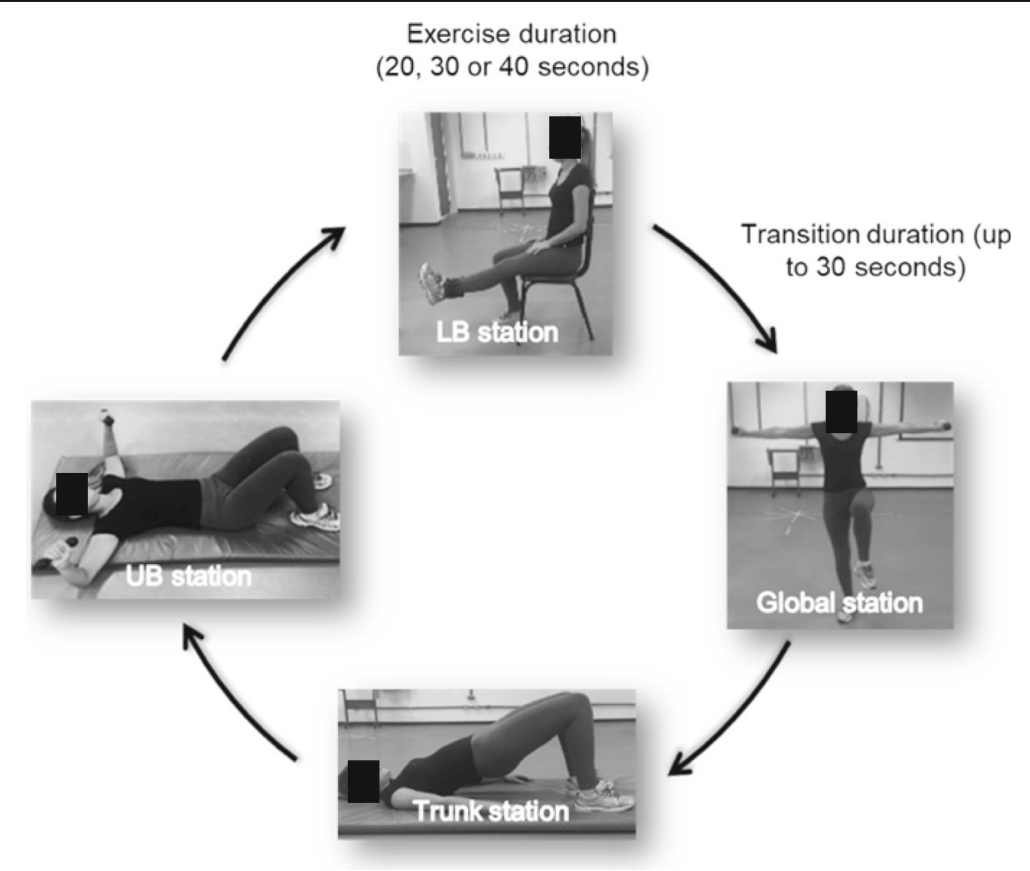

Fig. 2 Circuit training protocol - Station organization 


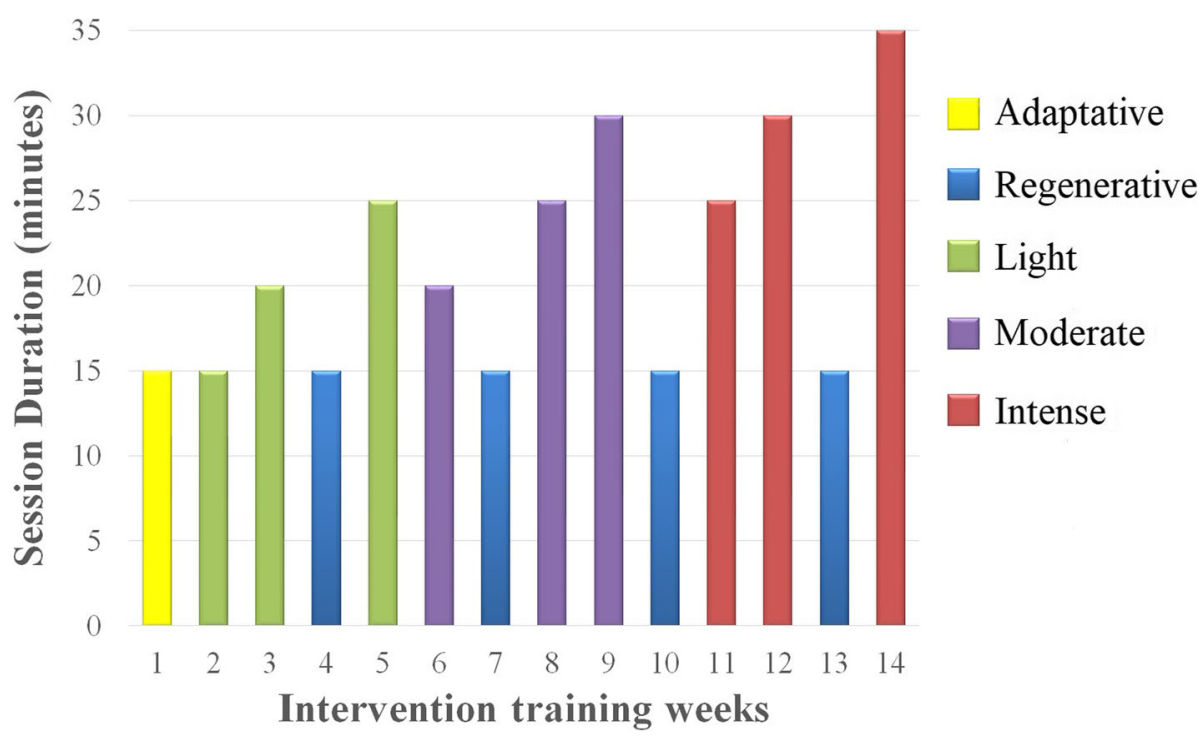

Fig. 3 Periodized dynamic loads of the circuit training protocol

The blue columns represent regenerative weeks in which the training will be performed at light intensity, but in this case, each exercise will have a $10 \mathrm{~s}$ duration with an increase of $10 \mathrm{~s}$ in the recovery time of each station, accounting for a smaller workload with mild exercise.

The exercise intensity will be increased at the 6 th and 11th weeks, as shown in Fig. 3. This will be based on the pilot study performed, as described.

It is possible to observe that from the second week, the protocol follows a progression method based on the intensity of effort and the volume of work (time). Three points must be observed in this model: $\mathrm{i}$-) the three intensities, according to the proposed classification, are carried out for three weeks each; ii) every two weeks of training, with intensities that aim at adaptation through stress, there is a recovery week (increase of metabolism with less structural and cardiovascular impact) and; iii) the two weeks between the recoveries are composed of implementation in the workload or in the effort intensity.

Thus, the planning progresses in a nonlinear manner. Changes in volume and intensity offer new challenges every week and show how it becomes increasingly easy to perform lower-intensity exercise. The principle of adaptation suggests stress and recovery to achieve goals and this was the intention in setting up this routine: To cause a controlled stress (stratification already described), to increment such stress, (either in the workload or the intensity of the effort) and to alternate periods of increment and recovery to stimulate the perception of adaptation acquired by participants [84].
Immediately after each training session, RPE; Final blood pressure (BPf); final heart rate (HRf); and VAS will be measured again.

\section{Strength training protocol}

Strength training is one of the most common types of exercise prescribed for the knee OA population, and in general has shown beneficial results on various parameters. For this reason, the strength training protocol used in this study will follow the model proposed by Selistre et al. (2017) with minor modifications. This protocol demonstrated reduced muscle weakness, pain, and disability in men with early knee osteoarthritis [81]. Each session will have an average duration of $60 \mathrm{~min}$, with three sessions per week for 14 weeks, also totaling 42 exercise sessions. The same exercises as performed in the circuit training protocol were selected for the lower body and trunk. The strength training will also include the same warm-up (five minutes of stationary bicycle) and cool-down strategies (5 min global stretching).

Table 1 shows the exercise distribution during the 14-week strength training protocol. The strength training protocol is divided into three levels. In the first level (1st-5th weeks), three quadriceps strengthening exercises and one strengthening exercise for the hamstrings, hip abductors, and adductors will be performed. In the second level (6th-9th weeks), three quadriceps strengthening exercises and strengthening exercise for the hamstrings, hip abductors, and adductors, and three trunk exercises will be performed. In the third level (11th-14th weeks), the same exercises as in the second level will be performed, but with higher difficulty. 
Table 1 14-week Strength Training Protocol exercise distribution

\begin{tabular}{|c|c|}
\hline Level/Weeks & Exercise \\
\hline \multirow[t]{6}{*}{ Level 1 - 1st to 5th weeks } & Semi squats $\left(50^{\circ}\right)^{\mathrm{a}}$ \\
\hline & $\begin{array}{l}\text { Knee extensor strengthening with ankle } \\
\text { weights. }{ }^{a}\end{array}$ \\
\hline & Straight leg raises with ankle weights. ${ }^{a}$ \\
\hline & $\begin{array}{l}\text { Hip abductor strengthening with ankle } \\
\text { weights. }^{{ }^{2}}\end{array}$ \\
\hline & $\begin{array}{l}\text { Hip adductor strengthening with ankle } \\
\text { weights. }\end{array}$ \\
\hline & $\begin{array}{l}\text { Knee flexor strengthening with ankle } \\
\text { weights. }\end{array}$ \\
\hline \multirow{4}{*}{$\begin{array}{l}\text { Level } 2 \text { - 6th to 9th } \\
\text { weeks }\end{array}$} & Level1 \\
\hline & Traditional bridge ${ }^{a}$ \\
\hline & Knee plank ${ }^{b}$ \\
\hline & Side knee plank ${ }^{b}$ \\
\hline \multirow{4}{*}{$\begin{array}{l}\text { Level } 3 \text { - 10th to } 14 \text { th } \\
\text { weeks }\end{array}$} & Level1 \\
\hline & Plank $k^{b}$ \\
\hline & Side plank ${ }^{\mathrm{b}}$ \\
\hline & Bridge on bosu ${ }^{b}$ \\
\hline
\end{tabular}

The initial load set for each exercise will be based on the one repetition maximum test (1 RM). Strengthening exercises will be performed in two sets of 15 repetitions, using 25\% 1RM for hip adductors and abductors, and $50 \% 1 \mathrm{RM}$ for the quadriceps and hamstrings, using ankle weights. Ankle weights will be progressed if necessary. During knee extensor and flexor, and hip adductors and abductors strengthening, participants will perform each repetition with a $5 \mathrm{~s}$ isometric muscle contraction. Exercises for the trunk will be performed in three 10-s series, increasing the duration when participants are able.

\section{Educational protocol}

The educational protocol will follow the model proposed by Messier et al. (2013b), in order to provide care, social interaction, and health education [2]. Meetings of 60 min will be held twice a month for 14 weeks, totaling 8 meetings. Interactive presentations will be performed addressing topics such as pathophysiology of osteoarthritis, and American College of Rheumatology (ACR) recommendations on nutrition, posture, and lifestyle. In addition, at the end of the meetings, participants will perform the same stretching exercise as performed in the exercised intervention protocols, but only on the upper body for five minutes, in order to improve adherence and increase the perceived benefits. According to the authors, there is no evidence that this practice of health education can directly influence the primary results of the present study [2]. Participants in the trained groups will also participate in the education and health sessions at separate times.

\section{Statistical analysis}

Data analysis will be performed using the software Statistical Package for the Social Sciences version 20.0 (SPSS Inc., Chicago, IL, USA). An intention to treat analysis will be conducted for all data analyses. Initially, descriptive analysis of variables will be performed as mean, standard deviation, and coefficient of variation. Next, normality and homogeneity of variance will be checked by the Kolmogorov-Smirnov test and Levene's test, respectively. If normal distribution of data is not observed, the same transformations will be carried out, to allow the application of parametric tests.

Two-way ANOVA (group X time) will be conducted for the dependent variables. When significant differences were observed in the group or interaction, will be performed a post-hoc Bonferroni Test. For all measured variables, the estimated sphericity will be examined in accordance with Mauchly's W Test, and the Greenhouse-Geisser correction will be used as needed. For comparison between moments, for uCTX-II, sCOMP, and MVIC repeated measures ANOVA (baseline X during protocols $\mathrm{X}$ follow-up) will be performed. When significant main effects are found, the Bonferroni test will be used to identify statistical differences. For IL1- $\beta$, IL-6, TNF- $\alpha$, leptin, adiponectin, WOMAC, PCS, DXA, computed tomography, and functional performance-based tests, the $\mathrm{t}$-test will be used (baseline $\mathrm{X}$ follow-up). For all analyzes a significance level of $5 \%$ will be adopted $(p<$ 0.05 ). No interim efficacy or subgroup analyses are planned.

\section{Discussion}

Despite the large number of studies investigating the effects of physical exercise on the knee OA population, there are few clinical trials that focus on altered thigh composition (i.e. increased adipose tissue and decreased muscle mass). No studies were found which investigated the effects of a periodized circuit training protocol in this population, based on intensity of specific effort for this population with a randomized controlled trial model. As previously mentioned, circuit training promotes changes in body composition and muscle strengthening, as well as being a training modality tolerated by patients with knee OA, as attested in our pilot study. This training model has already been used in several other chronic dysfunctions and should be better explored considering its diverse benefits, both in skeletal muscle and the cardiorespiratory system.

In addition to the physical benefits, it is worth emphasizing the financial benefits that this modality provides, considering the greater number of participants in the 
same session, shorter session duration, and the possibility of performing the exercises without needing machines, commonly used in strength training.

Given the lack of information in the published literature and the public health impact that exercise intervention protocols can provide, the effects of periodized circuit training on thigh composition (adipose tissue and muscle mass), pain, functional performance, inflammation, and degeneration biomarkers are critical to delineate.

This study follows the OARSI recommendations for designing clinical trials for patients with knee OA [48] as well as the CONSORT recommendations for performing randomized controlled trials [54]. In addition, this study also has as a differential a control group, based on the model proposed by Messier et al. 2013. This educational protocol comparison group provides attention, social interaction, and health education. The purpose of including this group is to encourage recruitment, adherence, and benefit, and not to influence the primary outcome directly: no evidence was found that suggests that health education alone can affect body composition, pain, muscle strength, functional performance, or biomarkers. Considering that older adults are less likely to attend if they think any treatment group does not provide personal benefit $[2,45]$, including a control group providing similar benefits, such as those typically offered by community health-education programs, seems to be a good way to improve the feasibility of the study.

Thus, considering the high prevalence and various disorders related to knee OA, as well as the gaps in the scientific literature about the ideal prescription of exercise training protocols, assessing the efficacy of a periodized circuit training protocol, with a design focused on intensity progression, and its impact on body composition, as well as on the various related outcomes, has immediate and high clinical impact. The results of this study will provide critically needed guidance to the health care system for the treatment and prevention of complications related to knee OA.

\section{Additional file}

Additional file 1: Circuit Training Protocol - Selected exercises descriptions. (DOCX 5584 kb)

\section{Acknowledgements}

The authors would like to thank the pilot study participants, and FAPESP and CAPES for the financial support to the investigators of this study.

\section{Funding}

This study is supported by the São Paulo Research Foundation (FAPESP process numbers 2016/05047-3 and 2013/00798-2) and the Coordination for the Improvement of Higher Education Personnel (CAPES) for graduate students' salaries.
Availability of data and materials

Not applicable, this manuscript does not contain any data, since it is a protocol.

\section{Authors' contributions}

ACA, CMP, and SMM conceptualized the circuit training protocol; ACA, MGP, and JBA conducted the pilot study. ACA, MGP, JBA, and SMM established the selection criteria; GHG was in charge of the sample size calculation, randomization, and data analyses conceptualization. All authors read and made comments on previous drafts of the manuscript and approved the final manuscript.

\section{Ethics approval and consent to participate}

This study was approved by the Research in Human Ethics Committee of the Federal University of São Carlos-SP. Participants will be informed about the procedures that will be performed throughout the research, and after agreeing to participate, will sign a free informed consent form (ICF). This study will be conducted according to Resolution 196/96 norms of the National Health Council on research involving humans.

\section{Consent for publication}

Written consent to publish the images was received from the person in Fig. 2 and in the supplemental material.

\section{Competing interests}

The authors declare that they have no competing interests.

\section{Publisher's Note}

Springer Nature remains neutral with regard to jurisdictional claims in published maps and institutional affiliations.

\section{Author details}

'Department of Physical Therapy, Federal University of São Carlos (UFSCar), Km 235,Rod. Washington Luís-SP310, Postal code, São Carlos, (SP) 13565-905, Brazil. 'Department of Physical Therapy, São Paulo State University (UNESP) School of Sciences and Technology, 305, Roberto Simonsen St., Presidente Prudente, (SP) 19060-900, Brazil.

Received: 29 January 2018 Accepted: 26 October 2018

Published online: 30 November 2018

\section{References}

1. Arden NK, Leyland KM. Osteoarthritis year 2013 in review: clinical. Osteoarthr Cartil [Internet]. 2013;21(10):1409-13.

2. Messier SP, Mihalko SL, Beavers DP, Nicklas BJ, Devita P, Carr JJ, et al. Strength Training for Arthritis Trial (START): design and rationale. BMC Musculoskelet Disord [Internet]. 2013;14(1):208.

3. Waller B, Munukka M, Rantalainen T, Lammentausta E, Nieminen MT, Kujala UM, et al. Effects of high intensity resistance aquatic training on body composition and walking speed in women with mild knee osteoarthritis : a 4-month RCT with 12-month follow-up. 2017:25:1238-1246.

4. Marcus RL, Addison O, Dibble LE, Foreman KB, Morrell G, Lastayo P. Intramuscular adipose tissue, sarcopenia, and mobility function in older individuals. J Aging Res. 2012;2012

5. Gerber C, Schneeberger AG, Hoppeler H, Meyer DC. Correlation of atrophy and fatty infiltration on strength and integrity of rotator cuff repairs: A study in thirteen patients. J Shoulder Elbow Surg. 2007;16(6):691-6.

6. Beasley LE, Koster A, Newman AB, Javaid MK, Ferrucci L, Kritchevsky SB, et al. Body composition measures from $\mathrm{CT}$ and inflammation. Obesity (Silver Spring). 2009;17(5):1062-9.

7. Maly MR, Calder KM, Maclntyre NJ, Beattie K. a. Relationship of intermuscular fat volume in the thigh with knee extensor strength and physical performance in women at risk of or with knee osteoarthritis. Arthritis Care Res. 2013:65(1):44-52.

8. Kumar D, Karampinos DC, MacLeod TD, Lin W, Nardo L, Li X, et al. Quadriceps intramuscular fat fraction rather than muscle size is associated with knee osteoarthritis. Osteoarthr Cartil [Internet]. 2014;22(2):226-34.

9. Addison $\mathrm{O}$, Marcus RL, Lastayo PC, Ryan AS. Intermuscular fat: a review of the consequences and causes. Int J Endocrinol. 2014;2014:34-6. 
10. Hao D, Li M, Wu Z, Duan Y, Li D, Qiu G. Synovial fluid level of adiponectin correlated with levels of aggrecan degradation markers in osteoarthritis. Rheumatol Int [Internet]. 2011;31(11):1433-7.

11. Collins KH, Herzog W, MacDonald GZ, Reimer RA, Rios JL, Smith IC, et al. Obesity, metabolic syndrome, and musculoskeletal disease: common inflammatory pathways suggest a central role for loss of muscle integrity. Front Physiol. 2018;9.

12. Hunt MA, Pollock CL, Kraus VB, Saxne T, Peters S, Huebner JL, et al. Relationships amongst osteoarthritis biomarkers, dynamic knee joint load, and exercise: results from a randomized controlled pilot study. BMC Musculoskelet Disord [Internet]. 2013;14(1):115.

13. Pearson MJ, Herndler-Brandstetter D, Tariq MA, Nicholson TA, Philp AM, Smith $\mathrm{HL}$, et al. IL-6 secretion in osteoarthritis patients is mediated by chondrocyte-synovial fibroblast cross-talk and is enhanced by obesity. Sci Rep. 2017;7(1):1-11.

14. Abella V, Scotece M, Conde J, Pino J, Gonzalez-Gay MA, Gómez-Reino $J$, et al. Leptin in the interplay of inflammation, metabolism and immune system disorders. Nat Rev Rheumatol [Internet]. 2017;5(13):100.

15. Stern JH, Rutkowski JM, Scherer PE. Adiponectin, leptin, and fatty acids in the maintenance of metabolic homeostasis through adipose tissue crosstalk. Cell Metab. 2016;23(5):770-84.

16. Mobasheri A, Bay-Jensen AC, van Spil WE, Larkin J, Levesque MC. Osteoarthritis year in review 2016: biomarkers (biochemical markers). Osteoarthr Cartil [Internet]. 2017;25(2):199-208.

17. Bay-Jensen AC, Reker D, Kjelgaard-Petersen CF, Mobasheri A, Karsdal MA Ladel C, et al. Osteoarthritis year in review 2015: Soluble biomarkers and the BIPED criteria. Osteoarthr Cartil [Internet]. 2016;24(1):9-20.

18. Kraus VB, Collins JE, Hargrove D, Losina E, Nevitt M, Katz JN, et al. Predictive validity of biochemical biomarkers in knee osteoarthritis: data from the $\mathrm{FNIH}$ OA biomarkers consortium. Ann Rheum Dis. 2017;76(1):186-95.

19. Loeser RF, Beavers DP, Bay-Jensen AC, Karsdal MA, Nicklas BJ, Guermazi A, et al. Effects of dietary weight loss with and without exercise on interstitial matrix turnover and tissue inflammation biomarkers in adults with knee osteoarthritis: the intensive diet and exercise for arthritis trial (IDEA). Osteoarthr Cartil [Internet]. 2017;25(11):1822-8.

20. Helmark IC, Mikkelsen UR, Borglum J, Rothe A, Petersen MCH, Andersen O, et al. Exercise increases interleukin-10 levels both intraarticularly and perisynovially in patients with knee osteoarthritis: a randomized controlled trial. Arthritis Res Ther. 2010;12(4):R126.

21. Fransen M, McConnell S, Harmer AR, Van der Esch M, Simic M, Bennell KL. Exercise for osteoarthritis of the knee: a Cochrane systematic review. $\mathrm{Br}$ J Sports Med [Internet]. 2015; bjsports-2015-095424.

22. Messier SP, Loeser RF, Miller GD, Morgan TM, Rejeski WJ, Sevick MA, et al. Exercise and dietary weight loss in overweight and obese older adults with knee osteoarthritis: the arthritis, diet, and activity promotion trial. Arthritis Rheum. 2004;50(5):1501-10.

23. Bennell KL, Hinman RS. A review of the clinical evidence for exercise in osteoarthritis of the hip and knee. J Sci Med Sport [Internet]. 2011;14(1):4-9.

24. Beavers DP, Beavers KM, Loeser RF, Walton NR, Lyles MF, Nicklas BJ, et al. The independent and combined effects of intensive weight loss and exercise training on bone mineral density in overweight and obese older adults with osteoarthritis. Osteoarthr Cartil [Internet]. 2014;22(6):726-33.

25. Juhl C, Christensen R, Roos EM, Zhang W, Lund H. Impact of exercise type and dose on pain and disability in knee osteoarthritis: a systematic review and meta-regression analysis of randomized controlled trials. Arthritis Rheumatol. 2014;66(3):622-36.

26. Malas FÜ, Özçakar L, Kaymak B, Ulaşli A, Güner S, Kara M, et al. Effects of different strength training on muscle architecture: clinical and ultrasonographic evaluation in knee osteoarthritis. PM R. 2013;5(8):655-62.

27. Frontera W, Reid K, Phillips E. Muscle fiber size and function in elderly humans: a longitudinal study. J Appl [Internet]. 2008;02111:637-42.

28. Beavers KM, Beavers DP, Newman JJ, Anderson AM, Loeser RF, Nicklas BJ, et al. Effects of total and regional fat loss on plasma CRP and IL-6 in overweight and obese, older adults with knee osteoarthritis. Osteoarthr Cartil [Internet]. 2015;23(2):249-56.

29. Olson TP, Dengel DR, Leon AS, Schmitz KH. Changes in inflammatory biomarkers following one-year of moderate resistance training in overweight women. Int J Obes. 2007;31(6):996-1003.

30. Nguyen C, Lefèvre-Colau MM, Poiraudeau S, Rannou F. Rehabilitation (exercise and strength training) and osteoarthritis: A critical narrative review. Ann Phys Rehabil Med. 2016;59(3):190-5.
31. Romero-Arenas S, Blazevich AJ, Martínez-Pascual M, Pérez-Gómez J, Luque AJ, López-Román FJ, et al. Effects of high-resistance circuit training in an elderly population. Exp Gerontol [Internet]. 2013;48(3):334-40.

32. Scopaz KA, Piva SR, Gil AB, Ms PT, Woollard JD, Oddis CV, et al. The effect of baseline quadriceps activation on changes in quadriceps strength after exercise therapy in subjects with knee osteoarthritis. Arthritis Rheum. 2009;61(7):951-7.

33. Bocalini DS, Lima LS, de Andrade S, Madureira A, Rica RL, dos Santos RN, et al. Effects of circuit-based exercise programs on the body composition of elderly obese women. Clin Interv Aging. 2012;7:551-6.

34. Balachandran A, Krawczyk SN, Potiaumpai M, Signorile JF. High-speed circuit training vs hypertrophy training to improve physical function in sarcopenic obese adults: A randomized controlled trial. Exp Gerontol [Internet]. 2014;60: 64-71.

35. Miller MB, Pearcey GEP, Cahill F, McCarthy H, Stratton SBD, Noftall JC, et al. The effect of a short-term high-intensity circuit training program on work capacity, body composition, and blood profiles in sedentary obese men: a pilot study. Biomed Res Int. 2014;2014.

36. Kim H, Kang C, Park H, Lee M. Effects of vitamin D supplementation and circuit training on indices of obesity and insulin resistance in T2D and vitamin D deficient elderly women. J Exerc Nutr Biochem. 2014;18(3):249-57.

37. Nordgren B, Friden C, Demmelmaier I, Bergstrom G, Lundberg IE, Dufour $A B$, et al. An outsourced health-enhancing physical activity programme for people with rheumatoid arthritis: exploration of adherence and response. Rheumatology [Internet]. 2014:1065-73.

38. Magrans-Courtney T, Wilborn C, Rasmussen C, Ferreira M, Greenwood L, Campbell B, et al. Effects of diet type and supplementation of glucosamine, chondroitin, and MSM on body composition, functional status, and markers of health in women with knee osteoarthritis initiating a resistance-based exercise and weight loss program. J Int Soc Sports Nutr [Internet]. 2011;8(1):8.

39. Hsu W, Hsu W, Shen W, Lin Z, Chang S, Hsu RW. Circuit training enhances function in patients undergoing total knee arthroplasty : a retrospective cohort study. J Orthop Surg Res. 2017:1-9.

40. Al-Khlaifat L, Herrington LC, Tyson SF, Hammond A, Jones RK. The effectiveness of an exercise programme on dynamic balance in patients with medial knee osteoarthritis: a pilot study. Knee [Internet]. 2016;23:849-56.

41. Ruhdorfer A, Wirth W, Dannhauer T, Eckstein F. Longitudinal (4 year) change of thigh muscle and adipose tissue distribution in chronically painful vs painless knees - data from the osteoarthritis initiative. Osteoarthr Cartil [Internet]. 2015;23(8):1348-56.

42. Conroy M, Kwoh K, Krishnan E, Nevitt M, Boudreau RM, Carbone LD, et al. Muscle strength, mass and quality in older men and women with knee osteoarthritis: findings form health. Aging and Body Composition Study. 2012;64(1):15-21.

43. Ikeda S, Tsumura H, Torisu T. Age-related quadriceps-dominant muscle atrophy and incident radiographic knee osteoarthritis. J Orthop Sci. 2005 Jan:10(2):121-6.

44. Larsen BA, Allison MA, Kang E, Saad S, Laughlin GA, Araneta MRG, et al. Associations of physical activity and sedentary behavior with regional fat deposition. Med Sci Sports Exerc. 2014;46(3):520-8.

45. Messier SP, Mihalko SL, Legault C, Miller GD, Nicklas BJ, DeVita P, et al. Effects of intensive diet and exercise on knee joint loads, inflammation, and clinical outcomes among overweight and obese adults with knee osteoarthritis: the IDEA randomized clinical trial. JAMA [Internet]. 2013; 310(12):1263-73.

46. Nicklas BJ, Chmelo E, Delbono O, Carr JJ, Lyles MF, Marsh AP. Effects of resistance training with and without caloric restriction on physical function and mobility in overweight and obese older adults : a randomized controlled trial. Am J Clin Nutr. 2015;101:991-9.

47. Jacobs JL, Marcus RL, Morrell G, Lastayo P. Resistance exercise with older fallers: its impact on intermuscular adipose tissue. Biomed Res Int. 2014;2014.

48. Fitzgerald GK, Hinman RS, Zeni J, Risberg MA, Snyder-Mackler L, Bennell KL. OARSI clinical trials recommendations: design and conduct of clinical trials of rehabilitation interventions for osteoarthritis. Osteoarthritis Cartilage [Internet]. 2015;23(5):803-14.

49. Messier SP, Callaha LF, Golightly YM, Keefe FJ. OARSI clinical trials recommendations: design and conduct of clinical trials of rehabilitation interventions for osteoarthritis. Osteoarthritis Cartilage [Internet]. 2015;23(5): 803-14.

50. Agha RA, Altman DG, Rosin D. The SPIRIT 2013 statement - defining standard protocol items for trials. Int J Surg [Internet]. 2015;13:288-91. 
51. Chan AW, Tetzlaff JM, Gøtzsche PC, Altman DG, Mann H, Berlin JA, et al. SPIRIT 2013 explanation and elaboration: guidance for protocols of clinical trials. BMJ. 2013;346:1-42.

52. Chan A, Tetzlaff JM, Altman DG, Laupacis A, Gøtzsche PC, Krleža-Jerić K, et al. SPIRIT 2013 statement : defining standard protocol items for clinical trials. Ann Intern Med. 2013;158(3):200-7.

53. Kloppenburg M, Maheu E, Kraus VB, Cicuttini F, Doherty M, Dreiser RL, et al. OARSI clinical trials recommendations: design and conduct of clinical trials for hand osteoarthritis. Osteoarthr Cartil [Internet]. 2015;23(5):772-86.

54. Schulz KF, Altman DG, Moher D, Group C. CONSORT 2010 Statement: updated guidelines for reporting parallel group randomised trials. BMJ Open. 2010:340.

55. Griessmann S, Dannhauer T, Hudelmaier M, Ruhdorfer A, Wirth W, Himmer $M$, et al. Effects of strength and endurance training intervention on thigh intermuscular and subcutaneous adipose tissue in sarkopenic women. Osteoarthr Cartil [Internet]. 2014;22(2014):S317.

56. Kellgren JH, Lawrence JS. Radiological assessment of osteo-arthrosis Ann Rheum Dis. 1957;16(3):494-503.

57. Hortobágyi T, Garry J, Holbert D, Devita P. Aberrations in the control of quadriceps muscle force in patients with knee osteoarthritis. Arthritis Rheum. 2004:51(4):562-9.

58. Zacaron K, Dias J, Abreu N, Dias R. Nível de atividade física, dor e edema e suas relações com a disfunção muscular do joelho de idosos com osteoartrite. Rev Bras Fisioter. 2006;10(3):279-84

59. Bhattacharya R, Kumar V, Safawi E, Finn P, Hui AC. The knee skyline radiograph: its usefulness in the diagnosis of patello-femoral osteoarthritis. Int Orthop. 2007:31:247-52.

60. Selistre LF, Mattiello SM, Nakagawa TH, Gonçalves GH, Petrella M, Jones RK.J Electromyogr Kinesiol. The relationship between external knee moments and muscle co-activation in subjects with medial knee osteoarthritis. J Electromyogr Kinesiol. 2017;33:64-72.

61. Altman RD, Gold GE. Atlas of individual radiographic features in osteoarthritis. Osteoarthr Cartil. 2007;15:1-56

62. Felson DT, Niu J, Guermazi A, Sack B, Aliabadi P. Defining radiographic incidence and progression of knee osteoarthritis: suggested modifications of the Kellgren and Lawrence scale. Ann Rheum Dis. 2011;70(11):1884-6.

63. Avila JJ, Gutierres JA, Sheehy ME, Lofgren IE, Delmonico MJ. Effect of moderate intensity resistance training during weight loss on body composition and physical performance in overweight older adults. Eur J Appl Physiol. 2010;109(3):517-25.

64. Goodpaster BH, Kelley DE, Thaete FL, He J, Ross R. Skeletal muscle attenuation determined by computed tomography is associated with skeletal muscle lipid content. J Appl Physiol. 2000;89(1):104-10.

65. Nana A, Slater GJ, Stewart AD, Burke LM. Methodology review : using dualenergy X-ray absorptiometry ( DXA ) for the assessment of body composition in athletes and active people current uses of DXA estimates of Int J Sport Nutr Exerc Metab. 2014;2014:198-215.

66. Nana A, Slater GJ, Hopkins WG, Burke LM. Effects of exercise sessions on DXA measurements of body composition in active people. Med Sci Sports Exerc. 2013;45(1):178-85.

67. Denning WM, Winward JG, Becker Pardo M, Hopkins JT, Seeley MK. Body weight independently affects articular cartilage catabolism. J Sports Sci Med [Internet]. 2015;14(2):290-6.

68. Reijman M, Hazes JMW, Bierma-Zeinstra SM, Koes BW, Christgau S, Christiansen C, et al. A new marker for osteoarthritis: cross-sectional and longitudinal approach. Arthritis Rheum. 2004;50(8):2471-8.

69. Marx FC, De Oliveira LM, Bellini CG, Ribeiro MCC. Tradução e validação cultural do questionário algofuncional de Lequesne para osteoartrite de joelhos e quadris para a língua Portuguesa. Rev Bras Reumatol. 2006;46(4):253-60.

70. Sullivan MJL, Bishop SR, Pivik J. The pain catastrophizing scale : development and validation. Psychol Assess. 1995;7(4):524-32.

71. Dobson F, Hinman RS, Roos EM, Abbott JH, Stratford P, Davis AM, et al. OARSI recommended performance-based tests to assess physical function in people diagnosed with hip or knee osteoarthritis. Osteoarthr Cartil [Internet]. 2013;21(8):1042-52.

72. McAlindon TE, Driban JB, Henrotin Y, Hunter DJ, Jiang G-L, Skou ST, et al. OARSI clinical trials recommendations: design, conduct, and reporting of clinical trials for knee osteoarthritis. Osteoarthritis Cartilage [Internet]. 2015; 23(5):747-60.

73. Hare KB, Lohmander LS, Christensen R, Roos EM. Arthroscopic partial meniscectomy in middle-aged patients with mild or no knee osteoarthritis: a protocol for a double-blind, randomized sham-controlled multi-centre trial. BMC Musculoskelet Disord [Internet]. 2013;14(1):71.

74. Clements S, Samuel D. Knee extensor strength measured using a Biodex dynamometer and an adapted hand held dynamometer. Int J Ther Rehabil [Internet]. 2014;21:274-8.

75. Martin HJ, Yule V, Syddall HE, Dennison EM, Cooper C, Aihie SA. Is handheld dynamometry useful for the measurement of quadriceps strength in older people? A comparison with the gold standard biodex dynamometry. Gerontology. 2006;52(3):154-9.

76. Martins J, da Silva JR, Da Silva MRB, Bevilaqua-Grossi D. Reliability and Validity of the Belt-Stabilized Handheld Dynamometer in Hip- and KneeStrength Tests. J Athl Train [Internet]. 2017;52(9):1062-6050-52.6.04.

77. Romero-Franco N , Jiménez-Reyes $P$, Montaño-Munuera JA. Validity and reliability of a low-cost digital dynamometer for measuring isometric strength of lower limb. J Sports Sci [Internet]. 2017;35(22):2179-84.

78. Manini TM, Buford TW, Lott DJ, Vandenborne K, Daniels MJ, Knaggs JD, et al Effect of dietary restriction and exercise on lower extremity tissue compartments in obese, older women: a pilot study. Journals Gerontol - Ser A Biol Sci Med Sci. 2014;69(1):101-8.

79. Ageberg E, Nilsdotter A, Kosek E, Roos EM. Effects of neuromuscular training (NEMEX-TJR) on patient-reported outcomes and physical function in severe primary hip or knee osteoarthritis: a controlled before-and-after study. BMC Musculoskelet Disord [Internet]. 2013;14(1):232.

80. Bennell KL, Kyriakides M, Metcalf B, Egerton T, Wrigley TV, Hodges PW, et al. Neuromuscular versus quadriceps strengthening exercise in patients with medial knee osteoarthritis and varus malalignment: a randomized controlled trial. Arthritis Rheumatol. 2014;66(4):950-9.

81. Selistre LFA, Gonçalves GH, Petrella M, Sato TO, Serrão PRMS, Vasilceac FA, Mattiello SM. The effects of strengthening, neuromuscular and lumbopelvic stabilization exercises on strength, physical function and symptoms in men with mild knee osteoarthritis : A pilot study. Isokinet Exerc Sci 2017;1:1-9.

82. Muyor JM. Exercise intensity and validity of the ratings of perceived exertion (Borg and OMNI scales) in an indoor cycling session. J Hum Kinet [Internet]. 2013;39(1):93-101.

83. Klika B, Jordan C. Circuit Training Using Body Weight : ACSM's Heal Fit J. 2013;17(3):8-13.

84. Turner A. The science and practice of periodization: a brief review. Strength Cond J [Internet]. 2011;33(2):91
Ready to submit your research? Choose BMC and benefit from:
- fast, convenient online submission
- thorough peer review by experienced researchers in your field
- rapid publication on acceptance
- support for research data, including large and complex data types
- gold Open Access which fosters wider collaboration and increased citations
- maximum visibility for your research: over $100 \mathrm{M}$ website views per year
At BMC, research is always in progress. 